\title{
Subtorr Operation of a Miniature Gas Ionization Sensor Based on Gold Nanowires
}

\author{
Ramin Banan Sadeghian*, Nicoleta Chivu and Mojtaba Kahrizi \\ Department of Electrical and Computer Engineering, Concordia University, \\ Montréal, QC, H4G2W1, Canada
}

(Received May 24, 2008; accepted September 8, 2008)

Key words: field enhancement, gas ionization sensors, gold nanowires, PIC/MCC

Vertically aligned freestanding gold nanowires (AuNWs) were synthesized and deposited on one of the electrodes of a capacitor-like gas ionization cell, whereas the counterelectrode was a polished silicon wafer coated with aluminum on both sides. The field enhancement property of high-aspect-ratio AuNWs was employed to reduce the gaseous breakdown voltage $\left(V_{\mathrm{b}}\right)$ at room temperature. The device was characterized in low-pressure air, and tested in subtorr argon where it demonstrated a considerable reduction in $V_{\mathrm{b}}$ compared with uniform field conditions and with its earlier counterparts. The dependences of $V_{\mathrm{b}}$ and prebreakdown currents on the polarity of applied voltage were studied. A particle-in-cell/Monte-Carlo-collision (PIC/MCC) model for the device was also developed to simulate the breakdown process within the same pressure range in which measurements were carried out. The simulated $V_{\mathrm{b}}-P$ curve showed good agreement with the measured characteristics.

\section{Introduction}

Of late, researchers have reported the fabrication of gas ionization sensors using carbon nanotube (CNT) arrays. ${ }^{(1-5)}$ These devices employ the high electric fields on sharp CNT tips to generate microdischarges. The unique discharge characteristics of each gas — breakdown voltage $\left(V_{\mathrm{b}}\right)$ and discharge current — can then be used to identify the gas type and measure the gas concentration. ${ }^{(3)}$ Therefore, ionization sensors offer enhanced selectivity and short response times over conventional nanostructured catalystbased devices. ${ }^{(6-9)}$

One of the inherent characteristics of CNT-based ionization sensors is the reduced overall field enhancement factor of closely packed CNTs owing to their small intertip distance, and accordingly large electrostatic interaction. In addition, extremely dense CNTs make dispersion of gas difficult, so the sensor cannot be reused prior to cleaning treatment. ${ }^{(10)}$

This report is part of ongoing research to develop a miniaturized low-voltage, lowpressure, and highly sensitive gas sensor. The preliminary results and fabrication of

*Corresponding author: e-mail: r_banan@ece.concordia.ca 
the sensor based on gold nanowires (AuNWs) were reported earlier.(11,12) In this paper, we report on the operation of the device, particularly in subtorr pressures. Because there is a general lack of analysis and numerical simulation in the literature, on the breakdown process in these types of sensor, we have developed a simulation model for the device. The results of simulations at subtorr pressures are also presented in this report. Our model, which is based on combined particle-in-cell and Monte-Carlocollision approaches (PIC-MCC), provides a platform for the further development and optimization of the device.

\section{Theory of Operation}

\subsection{Gaseous discharge and breakdown}

Depending on applied DC voltage, there are three distinct regimes in the discharge $I-V$ characteristic of a parallel-plate structure before the breakdown of gas occurs: At low voltages, the discharge current $(I)$ depends on the velocity of already existing carriers, which is nearly proportional to the applied voltage $V$. Therefore, the device shows an ohmic conductance. At higher voltages, when all of the carriers generated in the gap arrive at the electrodes, $I$ saturates and becomes independent of $V$. The current at the anode in the saturation regime is limited by the rate of carrier production and is given by

$$
I_{\mathrm{a}(\mathrm{sat})}=e A d \frac{d n_{e}}{d t},
$$

where $e$ is the electron charge, $A$ is the electrode area, $d$ is the gap spacing, and $d n_{e} / d t$ is the rate of radiation-induced electron production in the gap. ${ }^{(13)}$ The ionic saturation current to the cathode is given by an identical expression, provided that the atoms are singly ionized.

If voltage is further increased, electrons make inelastic ionizing collisions with neutral gas molecules. In this regime, $I$ rises at an increasing rate until the gas breaks down at $V_{\mathrm{b}}$. The characteristic between the saturation and breakdown represents the third prebreakdown discharge regime, referred to as the Townsend discharge. The anode current in the Townsend discharge regime is given by

$$
I_{a}=\frac{I_{\mathrm{a}(\mathrm{sat})} e^{\alpha d}}{1-(\omega / \alpha)\left(e^{\alpha d}-1\right)},
$$

where $\alpha$, the Townsend primary ionization coefficient, represents the number of ionizing collisions an electron makes by moving $1 \mathrm{~cm}$ in the field direction, and, $\omega / \alpha$, the secondary ionization coefficient, represents the number of secondary electrons produced per primary ionization. Indeed, $\alpha$ and $\omega / \alpha$ are both functions of the reduced field $(E / N$ or $E / P)$, where $N$ and $P$ are the gas concentration and pressure, respectively. As a result, increasing $V$ could affect $\alpha$ and $\omega / \alpha$ in such a way that the denominator of eq. (2) becomes zero at $V_{\mathrm{b}}$. At that point, current would rise sharply and become self-sustained. ${ }^{(13,14)}$ Note that $\omega / \alpha$ is the sum of individual coefficients representing the 
various secondary ionization processes, from which the number of electrons liberated per incident positive ion, $\gamma$, is usually predominant. ${ }^{(14)}$ The Townsend breakdown theory largely applies to moderate-to-low-pressure gases.

Because breakdown ionization sensors use $V_{\mathrm{b}}$ as a measure of the gas type, it is essential that the dependence of $V_{\mathrm{b}}$ on $P$ be minimal. Paschen's law states that $V_{\mathrm{b}}$ is a function of the product $N \cdot d$, or $P \cdot d$ if a constant temperature is stipulated.(13,14) However, in inhomogeneous field distributions such as those at the vicinity of sharp tips, the curves of $V_{\mathrm{b}}$ vs. $P \cdot d$, for $P \cdot d>(P \cdot d)_{\min }$, become less steep as compared with those under uniform field conditions. These curves also tend to become flat as the gap spacing shrinks, because the product $P \cdot d$ becomes small in a wider pressure range. That is, the effect of $P$ on $V_{\mathrm{b}}$ becomes less at smaller gaps. In addition, the sensitivity of an ionization sensor can be related to $\left(V_{\mathrm{b}}\right)_{\min }$, in the sense that a more sensitive device is expected to detect lower gas concentrations with a smaller $V_{\mathrm{b}}$.

\subsection{Field enhancement effect}

It is known that high-aspect-ratio protrusions facing a flat electrode provide locally enhanced electric fields at their tip apexes, given by

$$
E_{\mathrm{loc}}=\beta E_{\mathrm{app}}
$$

where $E_{\text {app }}$ is the applied electric field defined as $V / d$ in parallel-plate configurations, and $\beta$ is the geometry-dependent field enhancement factor that usually increases with protrusion aspect ratio and tip sharpness. ${ }^{(15,16)}$ The contribution of field enhancement to $V_{\mathrm{b}}$ will be addressed later in $\S 6.2$.

\section{Selectivity Versus Gap Size}

The improved selectivity of CNT-based ionization sensors was mainly achieved at atmospheric pressure levels. ${ }^{(1-5,10)}$ Qualitative explanations on how the breakdown voltage would increase at lower pressures were given according to Paschen's law, which does not always hold true.(13,14) At low pressures or when the gap size $(d)$ is too small, there is a small number of gas molecules involved in the ionization process. Usually, in this case, $V_{\mathrm{b}}$ increases because the probability of breakdown decreases. At micrometer gap distances, on the other hand, the breakdown process is known to be governed by field emission and field evaporation from the peaks at the cathode and anode surfaces, respectively, which is very similar to vacuum conditions for larger gaps. ${ }^{(17,18)}$ Because both field emission and field evaporation are very strong functions of electric field, $V_{\mathrm{b}}$ may decrease with decreasing $d$. As a result, $V_{\mathrm{b}}$, the figure-of-merit of the sensor, would be less dependent on the type of the residing gas molecule, i.e., the selectivity of the sensor would be adversely affected. It is thus, imperative to maintain a reasonable $d$ while designing a miniature ionization sensor. Our suggested value for $d$ is $\sim 100 \mu \mathrm{m}$ or above, from which we have obtained very good selectivity. ${ }^{(11)}$ 


\section{Experimental Methods and Modeling}

\subsection{Preparation of freestanding gold nanowires}

In this work, AuNWs were grown by the template-assisted electrochemical method. Anopore $^{\circledR}$ anodic aluminum oxide (AAO) membranes from Whatman Co. (Anodisc 25) were used as templates. These membranes are $60 \mu \mathrm{m}$ thick and are formed by unconnected cylindrical pores with an average diameter of $2 r=180 \mathrm{~nm}$ and a separation of $s=300 \mathrm{~nm}$, which take up nearly $58 \mu \mathrm{m}$ of the thickness. The remaining $2 \mu \mathrm{m}$ at the bottom side contains interconnected pores of smaller size. An annular polypropylene ring is peripherally bonded to the membrane for ease of handling.

The bottom side of the Anopore membrane was sputter-coated with a 100-nmthick layer of silver to provide a conductive surface for nucleation of AuNWs. Because this layer does not plug the pores completely, electrodeposition takes place from both sides of the membrane. Nanowires were grown galvanostatically in a custom-made electrochemical microcell similar to that used in earlier works (current density $=0.32$ $\mathrm{mA} \cdot \mathrm{cm}^{-2}$, time $\left.=22 \mathrm{~h}\right) .{ }^{(12,19)}$ A platinum gauze, an $\mathrm{Ag} / \mathrm{AgCl}$ reference electrode, and the silver-coated side of the template served as the auxiliary, reference, and working electrodes, respectively. The membrane was then attached from the bottom side to a silicon wafer initially coated with titanium $(10 \mathrm{~nm})$ as an adhesive layer, and silver $(100 \mathrm{~nm})$ next. AAO was then dissolved in $2 \mathrm{M} \mathrm{NaOH}$ solution, leaving freestanding AuNWs. The morphology of the AuNW film was examined by field emission scanning electron microscopy (FESEM). Figure 1 shows an FESEM micrograph of the nanowire film surface. The substrate is tilted $\left(45^{\circ}\right)$ to expose the length of the wires. It can be seen that the AuNWs have a broad length distribution, lying in the range of $3 \mu \mathrm{m} \leq h \leq$ $16 \mu \mathrm{m}$. Note that because of the $45^{\circ}$ tilt, the actual lengths are $2^{1 / 2}$ times the observed lengths. The inset of Fig. 1 shows the bottom side of the template after sputter-coating with silver. It is clear that the pores are not completely blocked. ${ }^{(12)}$ Assuming replication takes place within all of the pores, the number of nanowires was estimated to be $3 \times 10^{9}$.

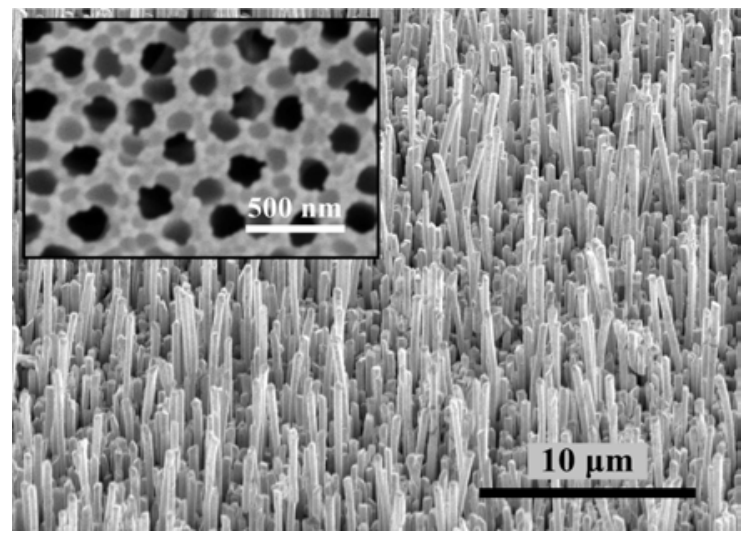

Fig. 1. FESEM micrograph of AuNW array after template removal. Inset: Bottom side of AAO after sputter-coating with Ag. 


\subsection{Sensor structure and measurement setup}

A schematic illustration of the device is shown in Fig. 2, where $s \approx 300 \mathrm{~nm}$ is the average spacing between the nanowires, and $d$ and $h$ are as defined earlier. After AAO was removed, the holding polypropylene ring (not shown here) was retained to serve as the spacer between the substrate and the counter electrode. Using the annular holding ring as the spacer is advantageous for two reasons: First, polypropylene is a dielectric with high insulating properties, ${ }^{(20)}$ and second, it eliminates the need for any deposition or micromachining processes. Slices were cut from the ring at some places to facilitate flow of gas into the gap. A double-side-polished highly doped P-type silicon wafer (1" diameter), as the counterelectrode, was then attached to the holding ring creating a gap spacing of $d \approx 120 \mu \mathrm{m}$. Before mounting the wafer, it was coated with aluminum on both sides by the physical vapor deposition technique, and annealed at $450^{\circ} \mathrm{C}$ for $30 \mathrm{~min}$ to form an exterior ohmic contact. The dimensions of the device are roughly $1.2 \mathrm{~mm} \times 5$ $\mathrm{cm}^{2}$.

The sensor was placed inside a vacuum chamber with electrical feedthroughs and air was pumped down to $10^{-5}$ Torr before each test was carried out. Then, the test gas was released into the chamber and the voltage was applied once the equilibrium pressures were achieved. The bottom electrode containing the nanowires and the flat counterelectrode were individually connected to two source-measure units (SMU). In this manner, the anode and cathode currents can be measured separately (See Fig. 2). We used two SMU channels of an HP4155 semiconductor parameter analyzer to conduct the pre-breakdown $I-V$ characterization, and two Keithley 2400 SMUs for $V_{\mathrm{b}}$ measurements.

\section{Modeling and Simulation}

We have developed a particle-in-cell-Monte-Carlo-collision (PIC-MCC) model for our device based on the XOOPIC simulator to simulate the discharge and breakdown

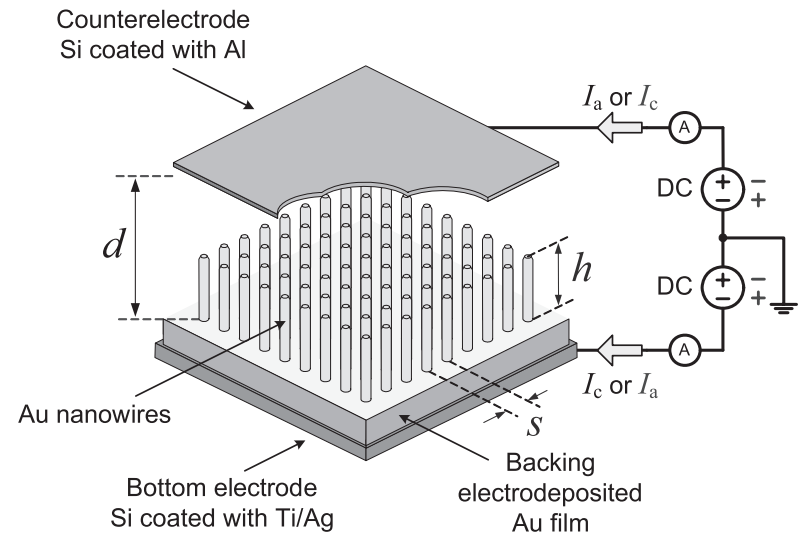

Fig. 2. Schematic illustration of the sensor, showing the definition of physical dimensions and the current to either electrode. $I_{\mathrm{c}}$ and $I_{\mathrm{a}}$ are the cathode and anode currents, respectively. 
processes and validate the experimentally obtained $V_{\mathrm{b}}-P$ characteristic. XOOPIC is designed to simulate plasmas in a well-defined confined geometry using a variety of boundary conditions. However, it lacks implantation of circuit elements such as the series resistance $(R)$ with the ionization cell. When driving the model with an ideal voltage source, very large currents could be drawn corresponding to increasing input power. With that positive feedback (increasing current via increasing conductivity of the plasma) it is very difficult to generate a DC discharge with an ideal voltage source. ${ }^{(21)}$ In an actual DC discharge, particularly at currents close to the breakdown point, the voltage drop across $R$ becomes important and must be considered. We have modified the code to introduce the effect of $R$. Our model also includes the contribution of field emission from AuNW tips to the discharge process. Note that simulation results are realistic only in the case of cathode AuNWs. For anode AuNWs, the electric breakdown is initiated by positive coronas at the AuNW tips. In a positive corona, electrons with high energy are concentrated close to the AuNW tip and the secondary ionization process is mainly photoionization in the gas, which XOOPIC does not support.

Since XOOPIC is a 2-D simulator, the nanowire array was modeled using parallel rectangular conductive sheets along the third imaginary dimension $(z)$ with unity length. Simulations were carried out at $P=0.02,0.05,0.1,0.5$, and 1 Torr. These pressure points lie within the range where the actual breakdown measurements were carried out. Before the bias was applied, at each pressure, it was assumed that a constant fraction of gas particles in the medium were singly ionized (singly) due to cosmic radiation. In addition to primary electrons, the contribution of secondary and field-emitted electrons to the discharge was considered. The secondary electrons were set to have the same collision model as the primary electrons; thus, the secondary ionization coefficient $(\omega / \alpha)$ was generated self-consistently. The AuNWs were defined here as both field-amplifying equipotential and field-emitter boundaries with a constant $\beta$. Field-emission current density from the AuNW tips was computed according to the general Fowler-Nordheim (FN) equation given as follows:

$$
J=\frac{A E_{\mathrm{loc}}{ }^{2}}{\phi_{\mathrm{Au}}} \exp \left(-\frac{B v \phi_{\mathrm{Au}}^{3 / 2}}{E_{\mathrm{loc}}}\right)
$$

where $A$ and $B$ are the universal FN constants, $\phi_{\mathrm{Au}}$ is the workfunction of gold, and $v$ is the field-dependent correction factor. ${ }^{(22,23)}$

At each pressure, the minimum voltage that led to formation of an avalanche from the nanowire tips was recorded as the corresponding $V_{\mathrm{b}}$. Figure 3 shows our 2-D model containing electrons of the first avalanche generated at $P=1$ Torr, $V=V_{\mathrm{b}}=-155 \mathrm{~V}$. The physical dimensions of the simulated regime and the definition of boundaries are specified in the figure.

Table 1 represents the parameters used in the simulation. $\Delta t$ is the time step calculated in accordance with Courant stability criteria.(24) $x$ and $y$ are the dimensions of the simulated device, $m$ and $n$ are the corresponding number of grids, and $\Delta x$ and $\Delta y$ are the spatial grid sizes. $n_{0}$ represents the percentage of gas particles already ionized. $n p 2 c$ is the number of physical particles per simulated (computational) particles, known as 


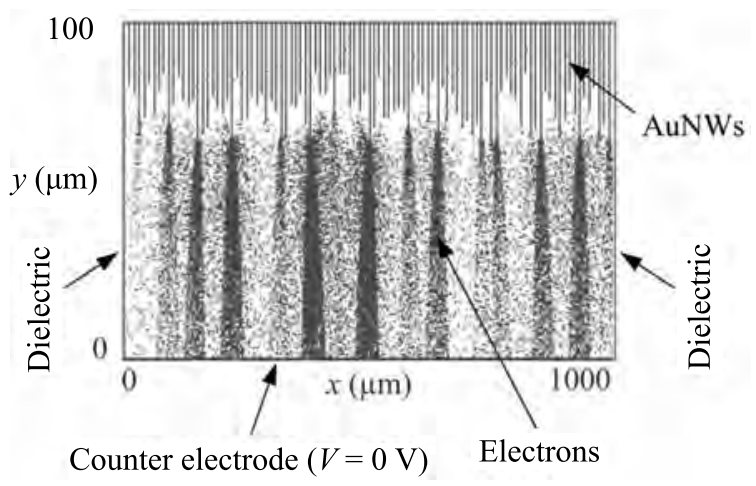

Fig. 3. 2-D model of the device showing electrons of an avalanche at $P=1$ Torr, $V=V_{\mathrm{b}}=-155 \mathrm{~V}$.

Table 1

Parameters used in our PIC-MCC discharge model.

\begin{tabular}{|c|c|c|c|c|c|c|c|}
\hline $\begin{array}{c}\text { cell } \\
\text { geometry }\end{array}$ & \multicolumn{3}{|c|}{$x, y=d, z$} & \multicolumn{2}{|c|}{$\begin{array}{c}\text { number of cells } \\
(\Delta x=x / m, \Delta y=y / n)\end{array}$} & \multicolumn{2}{|r|}{$\begin{array}{l}l_{\text {AuNW }} \\
(\mu \mathrm{m})\end{array}$} \\
\hline Cartesian & \multicolumn{3}{|c|}{$1 \mathrm{~mm}, 120 \mu \mathrm{m}, 1 \mathrm{~m}$} & \multicolumn{2}{|c|}{$m=300, n=100$} & \multicolumn{2}{|r|}{$25 \pm 10$} \\
\hline$V_{\mathrm{AuNW}}$ & $\varphi_{\mathrm{Au}}$ & & $\beta$ & $R$ & $P$ (Torr) & & $n_{0}(\%)$ \\
\hline $\begin{array}{c}-140--200 \mathrm{~V} \\
(-5 \mathrm{~V} \text { steps })\end{array}$ & $4.8 \mathrm{eV}$ & & 500 & $10 \Omega$ & $0.02-1$ & & $3 \times 10^{-7}$ \\
\hline$n p 2 c$ & $\Delta t(s)$ & $\Delta t_{\mathrm{i}} / \Delta t$ & $\gamma$ & $U_{\gamma}$ & $U_{\gamma \text {-max }}$ & $r_{\mathrm{K}}$ & $\varepsilon_{\mathrm{r}}$ \\
\hline $7.6 \times 10^{9}$ & $3.2 \times 10^{-14}$ & 10 & 1.2 & $12.5 \mathrm{eV}$ & $20 \mathrm{eV}$ & 0.5 & 1.0 \\
\hline
\end{tabular}

the superparticle ratio. $\Delta t_{\mathrm{i}} / \Delta t$ is the ion subcycle, $\gamma$ is the secondary electron factor per incident positive ion at the cathode, $U_{\gamma}$ is the threshold ion energy required for secondary electron emission, $U_{\gamma}$-max is the maximum allowed energy of the secondary electron, $r_{\mathrm{k}}$ is the dielectric reflection factor, and $\varepsilon_{\mathrm{r}}$ is the dielectric constant of the walls. For further explanation of the PIC-MCC parameters and definition of boundary condition, the reader may refer to ref. 23.

\section{Results and Discussion}

\subsection{Prebreakdown current}

The prebreakdown discharge current of the device at a low pressure $\left(10^{-5}\right.$ Torr $)$ of air was measured by performing a stepwise voltage sweep $(\Delta t=1 \mathrm{~s}, \Delta V=1 \mathrm{~V})$ up to \pm $200 \mathrm{~V}$. The appropriate sweep parameters were chosen so that the current due to the device capacitance could be neglected. Figure 4 shows the $I-V$ curves of both electrodes 


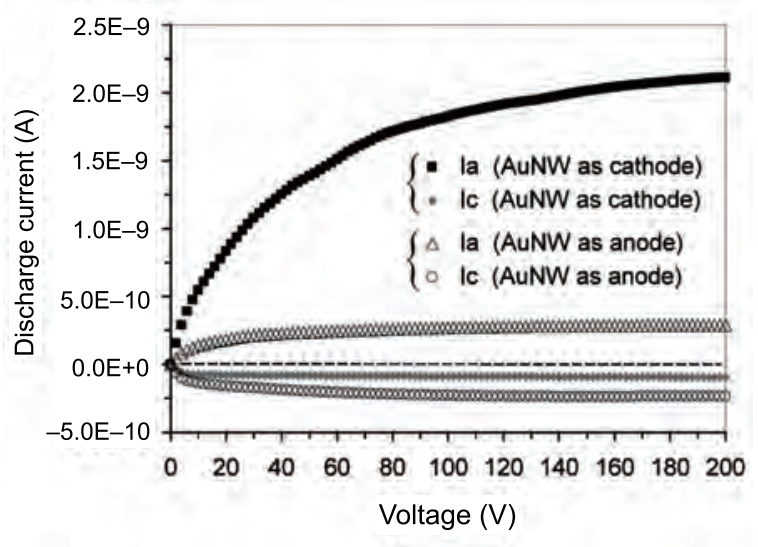

Fig. 4. Prebreakdown discharge current of both electrodes of the sensor in low-pressure air $(P=$ $10^{-5}$ Torr, $T=22^{\circ} \mathrm{C}$ ), for different AuNW film polarities. $I_{\mathrm{a}}$ is markedly higher when the AuNWs are at the cathode because of the enhanced ionization of low-pressure gas caused by repulsion of electrons from the nanowire tips.

(anode and cathode), with two different polarity configurations, as defined in Fig. 2. Operating the sensor in the prebreakdown regime gives us an insight into the ionization mechanisms in our particular configuration, i.e., nonuniform fields generated by AuNWs.

When AuNWs are configured as the anode, the saturation currents of both electrodes, $I_{\mathrm{c}(\text { sat)}}$, and $I_{\mathrm{a} \text { (sat) }}$, have nearly the same magnitude. This is similar to the case of saturation in uniform fields, where there is no electron impact ionization in the gap and the currents remain voltage-independent until Townsend discharge commences at higher voltages.

Evidently, the anode current $I_{\mathrm{a}}$, is markedly higher when the AuNW film is configured as the cathode. In addition, $I_{\text {a (sat) }}$ tends to slightly increase with $V$ before breakdown occurs. We believe this behavior can be attributed to abnormal repulsion of secondary electrons from the AuNW tips that trigger non-self-maintained ionization in low-pressure gas.

Electrons in the vicinity of sharp tips gain sufficient ionizing energies, but since the average electric field along the gap is not sufficient, the discharge is not self-sustained yet. Hence, the prebreakdown electron generation rate $d n_{e} / d t$ in eq. (1) is partly due to external radiation at the cathode, and partly due to ionization of neutrals by electron impact, the latter being field-dependent. In uniform fields, electron impact ionization normally occurs at higher voltages and leads to a self-sustained discharge as described by eq. (2).(13)

The observed lower cathode current $\left(I_{\mathrm{c}}\right)$ from the AuNWs is probably due to the higher workfunction of gold than that of aluminum. As a result, for the same amount of radiation, cathode emission from the AuNWs is smaller than emission from the aluminum-coated flat electrode. 


\subsection{Breakdown voltage}

The breakdown voltage of argon was measured in the pressure range of $0.01<P$ $<1$ Torr, at room temperature (corresponding to $5.4 \times 10^{-7}<N<5.4 \times 10^{-5} \mathrm{~mol}^{-1}$ liter $^{-1}$ ). After the gas pressure inside the chamber was stabilized, voltage was swept in the same manner as in the prebreakdown current measurements. The experiments were carried out with the AuNW film as the cathode and anode separately. Figure 5 shows the $V_{\mathrm{b}}-P$ characteristics of the device for both AuNW polarities. It was observed that $V_{\mathrm{b}}$ is considerably lower than typical breakdown voltages of argon in uniform fields under the same conditions. ${ }^{(16,25)}$ Locally, high electric fields at AuNW tips, promote ionization of gas at a lower applied field by affecting $\alpha$ and $\gamma$. However, reduction of $V_{\mathrm{b}}$, is not expected to be proportional to $\beta$, because although the nanowires have a wide length distribution, their tips still take up only a small fraction of the gap volume. The spread of the amplified field $\left(E_{\mathrm{loc}}\right)$ is therefore limited to a short distance. Also, as shown in Fig. 5, within the pressure range studied in this report, $V_{\mathrm{b}}$ was noticeably lower when the AuNWs were at the cathode. In this case, field-emitted electrons from the tips hasten formation of the avalanche, causing the breakdown to occur at lower voltages. As described earlier, due to the small gap size, $V_{\mathrm{b}}$ becomes independent of pressure at $P>0.2$ Torr for cathode AuNWs, and at $P>0.35$ Torr for anode AuNWs. Below $P \approx 0.2$ Torr, $V_{\mathrm{b}}$ tends to increase due to the reduction in number of diffusing gas particles into the gap.

No major deviation from the initial $V_{\mathrm{b}}-P$ curve shown in Fig. 5 was observed after repeating the test. This confirms the observation that the AuNWs have not undergone any geometrical degradation that may lead to a permanent reduction of the fieldenhancement effect. Also, the ionization and breakdown processes have not been affected by irreversible adsorption of gas atoms, usually observed in CNT-based sensors. ${ }^{(4,10)}$

Compared with the CNT-film ionization sensor fabricated by Modi et al., ${ }^{(3)}$ our device demonstrated a noticeably lower $V_{\mathrm{b}}$ for argon (162 V versus $220 \mathrm{~V}$ ), because even though individual CNTs, owing to their higher aspect ratios, may provide higher values of $\beta$, the

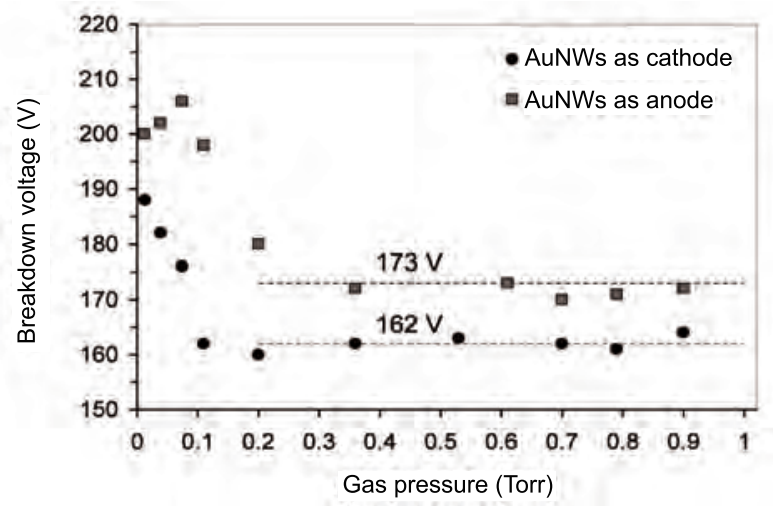

Fig. 5. $\quad V_{\mathrm{b}}-P$ characteristics of the GIS measured in subtorr argon at room temperature. $V_{\mathrm{b}}$ was unaffected by pressure for $P>0.2$ Torr, where the mean values were $173 \mathrm{~V}$ for anode AuNWs and $162 \mathrm{~V}$ for cathode AuNWs. $V_{\mathrm{b}}$ increased for $P<0.2$ Torr. 
effective value of $\beta$ is reduced owing to their small tip-to-tip distances (e.g. $s=50 \mathrm{~nm}^{(3)}$ ). AuNWs on the other hand, are sufficiently sparse that the interaction between the tips does not affect $\beta$.

Figure 6 shows the simulated $V_{\mathrm{b}}-P$ curve obtained in the pressure range of $0.02 \leq P$ $\leq 1$ Torr, at room temperature, with cathode AuNWs. At each pressure, the minimum voltage that generated an avalanche was recorded as $V_{\mathrm{b}}$. The error bars indicate that a voltage step of $5 \mathrm{~V}$ was used. Simulations show that electron avalanches are formed only on top of the longest nanowires, as short ones do not contribute much to the discharge (see Fig. 3). Electric fields on top of longer nanowires are further amplified because of charge separation, which in turn confines the avalanche. The similarity between the simulation and experimental results is discernable and proves the validity of our model.

Figure 7 shows the evolution of carriers with time at two extreme pressures, after $V_{\mathrm{b}}$ was applied. The arrows indicate depletion of already existing radiation-generated electrons during the first few picoseconds. The current flow afterwards is mainly due to the field-emitted electrons until argon ions gain sufficient energy to liberate secondary electrons upon collision with AuNW tips. Secondary electrons not only compensate for electron loss at boundaries, but also trigger ionizations at the space close to the tips, where avalanches are initiated. The higher population of electrons, compared with population of ions, at either pressure, shows that field-emitted electrons contribute to the formation of an avalanche. No restriction was applied to the voltage that could drop across $R$ in our simulated model. As a result, when the product $I \cdot R$ exceeded the applied voltage, the polarity of the ionization cell voltage would be reversed and consequently, the avalanche would extinguish. The turning points encircled in Figs. 7(a) and 7(b) indicate the reversal of cell voltage, after which the carriers are absorbed at the electrodes. The field emission component of the electron current is more clearly observed at low pressures (Fig. 7(b)); the plateaus in the curve of total number of electrons correspond to the periods where electron field emission dominates and the current is therefore constant. Note that at lower voltages $\left(V<V_{\mathrm{b}}\right)$, initially ionized particles subside until the gap is completely depleted.

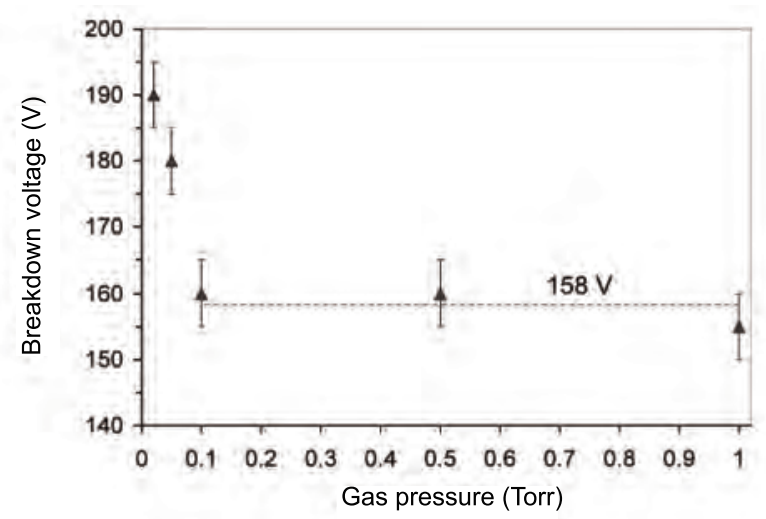

Fig. 6. Simulated $V_{\mathrm{b}}-P$ curve for AuNWs at the cathode. The simulated characteristic is close to the experimental characteristic. 

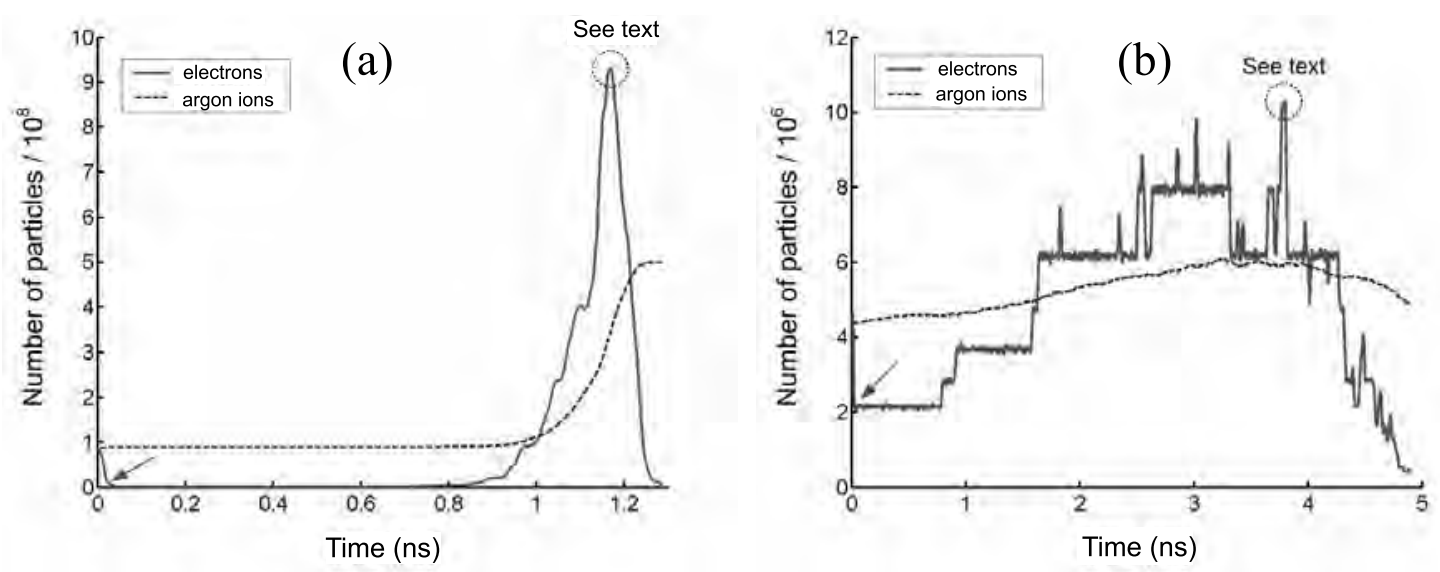

Fig. 7. Total number of electrons and ions in the gap vs time at two different pressures after $V_{\mathrm{b}}$ was applied to the AuNWs; (a) $P=1$ Torr, $V_{\mathrm{b}}=-155 \mathrm{~V}$, and (b) $P=0.05$ Torr, $V_{\mathrm{b}}=-180 \mathrm{~V}$. Input parameters are listed in Table 1.

\section{Conclusion}

An array of vertically aligned AuNWs was fabricated by galvanostatically filling the pores of nanoporous AAO templates, and then dissolving the template in a basic solution. The holding ring of the template was used as a spacer to create a double electrode gas ionizing cell using the AuNWs as field intensifiers. The above process proved that the fabrication of a highly sensitive ionization sensor can be achieved easily. The low-pressure prebreakdown discharge current of the device was measured at both of the electrodes and showed polarity dependence. Due to enhanced ionization caused by repulsion of electrons from the tips, $I_{\mathrm{a}}$ was one order of magnitude higher than $I_{\mathrm{c}}$ with the AuNWs as the cathode, while for anode AuNWs, $I_{\mathrm{a}}$ and $I_{\mathrm{c}}$ were almost equal. The device was able to detect subtorr argon with breakdown voltages considerably lower than those observed in devices with simple parallel-plate geometries. $V_{\mathrm{b}}$ was noticeably low with the AuNWs as the cathode because of the contribution of electron field emission.

The breakdown characteristic of the device for negative AuNWs was modeled using XOOPIC software. The simulated $V_{\mathrm{b}}-P$ curve was in good agreement with the experimental results.

This research has demonstrated the use of freestanding AuNWs as a replacement for CNTs in fabrication of miniature ionization sensors. The array of AuNWs displayed a larger increase in electric field on average, by further decreasing gaseous breakdown voltage, as compared with CNT-based ionization sensors with the same electrode separation. With the provision of monolithic integration of the detector with required microelectronics at the same substrate, the device could be the first integrated ionization detector of its own kind. 


\section{Acknowledgments}

This work was partially financially supported by the Natural Sciences and Engineering Research Council of Canada (NSERC) and by the Faculty of Engineering and Computer Science of Concordia University. The authors would like to thank Dr. Hojatollah Vali from the Department of Mining, Metals and Materials Engineering, McGill University, for providing SEM facilities for this project.

\section{References}

1 Z. Hou, J. Wu, W. Zhou, X. Wei, D. Xu, Y. Zhang and B. Cai: IEEE Trans. Electron Devices 54 (2007) 1545.

2 G. Hui, L. Wu, M. Pan, Y. Chen, T. Li and X. Zhang: Meas. Sci. Technol. 17 (2006) 2799.

3 A. Modi, N. Koratkar, E. Lass, B. Wei and P. M. Ajayan: Nature 424 (2003) 171.

4 Y. Zhang, J. Liu, X. Li, J. Dou, W. Liu, Y. He and C. Zhu: IVMC (IEEE, 2001) p. 13.

5 S. Kim: Sensors 6 (2006) 503.

6 J. Kong, N. R. Franklin, C. Zhou, M. G. Chapline, S. Peng, K. Cho and H. Dai: Science 287 (2000) 622.

7 Z. Liu and P. C. Searson: J. Phys. Chem. B 110 (2006) 4318.

8 Z. Fan, D. Wang, P.-C. Chang, W.-Y. Tseng and J. G. Lu: Appl. Phys. Lett. 85 (2004) 5923.

9 Z. Liu, T. Yamazaki, Y. Shen, T. Kikuta, N. Nakatani and T. Kawabata: Appl. Phys. Lett. 90 (2007) 173119.

10 Y. Zhang, J. Liu, X. Li and C. Zhu: Sens. Actuators, A 128 (2006) 278.

11 R. Banan-Sadeghian and M. Kahrizi: Sens. Actuators, A 137 (2007) 248.

12 R. Banan-Sadeghian and M. Kahrizi: ISIE (IEEE, Vigo, Spain, 2007) p. 1387.

13 A. M. Howatson: An introduction to gas discharges, 2nd ed (Pergamon Press, Oxford, 1976).

14 J. M. Meek and J. D. Craggs: Electrical Breakdown of Gases (John Wiley \& Sons, New York 1978).

15 R. G. Forbes, C. J. Edgcombe and U. Valdre: Ultramicroscopy 95 (2003) 57.

16 R. Banan-Sadeghian and M. Kahrizi: COMSOL Multiphysics Conf. 2005 (COMSOL, Boston, 2005) p. 251.

17 P. G. Slade and E. D. Taylor: IEEE Trans. Compon. Packag. Technol. 25 (2002) 390.

18 N. Zouache and A. Lefort: IEEE Trans. Dielect. Elect. Insul. 4 (1997) 358.

19 M. J. Tierney and C. R. Martin: J. Phys. Chem. 93 (1989) 2878.

20 C. Rodehed, A. Gustafsson and U. W. Gedde: IEEE Trans. Elect. Insul. 25 (1990) 1066.

21 J. Verboncoeur: OOPIC users digest, Vol. 50, Issue 3, Re: Electrode Circuit Current Measurement, Recipient: L. Weber (Tech-X Corporation, 2007).

22 R. Gomer: Field emission and Field ionization (Harvard Univ. Press, Cambridge, 1961).

23 OOPIC Pro User's Guide (Tech-X Corporation, Boulder 2006).

24 S. Mahalingam and J. A. Menart: Computational Model Tracking Primary Electrons, Secondary Electrons, and Ions in the Discharge Chamber of an Ion Engine (NASA 41st Joint Propulsion Conf. and Exhibit, Tucson, 2005).

25 M. Ali Hassouba, F. Fahmy Elakshar and A. Abuwali Garamoon: Fizika A 11 (2003) 81.

26 G. Petraconi, H. S. Maciel, R. S. Pessoa, G. Murakami, M. Massi, C. Otani, W. M. I. Uruchi and B. N. Sismanoglu: Braz. J. Phys. 34 (2004) 1662. 